\title{
Effects of Secondhand Smoke Exposure on Hearing and Auditory Evoked Potentials, ABR and AMLR in Young Adults
}

DOI: $10.3766 /$ jaaa.16161

\author{
Ishara Ramkissoon*† \\ Mihika Batavia
}

\begin{abstract}
Background: Population health is impacted by environmental secondhand smoke (SHS) exposure. Although the negative health effects of SHS exposure include respiratory problems in children (nonsmokers) as seen in cigarette smokers, other health impacts such as sensory function are not assumed to be the same for both passive nonsmokers and smokers. However, hearing loss was recently reported in adolescents and aging adults with SHS exposure, suggesting that SHS might impact auditory function similarly to cigarette smoking. Specific effects of SHS exposure on the central auditory system have not been fully described.
\end{abstract}

Purpose: To measure auditory function via pure-tone audiometry and evoked potentials in young nonsmoking adults aged 18-23 yr who reported exposure to environmental SHS.

Research Design: Participants were selected for the SHS-exposed (SHS-E) group first, followed by age and gender matched individuals for the SHS-unexposed (SHS-U) group. Self-reported nonsmoker status was confirmed by biochemical analysis of urine for cotinine level.

Study Sample: Potential participants $(\mathrm{N}=208)$ completed a questionnaire about health, smoking history, SHS exposure, and hearing ability. Individuals with any neurological conditions, alcohol/drug dependencies, excessive noise exposure, using certain medications, or current smokers were excluded. Twenty-two nonsmokers in excellent health consented to participate. Participants in the SHS-E group reported SHS exposure in home, work, or social settings for an extensive time period. Participants in the SHS-U group did not live with smokers and reported no SHS exposure, medication use, tinnitus, or any chemical exposures. Statistical analysis was conducted on data from 20 participants, 10 per group with a mean age of $20 \mathrm{yr}$.

Data Collection and Analysis: Participants underwent auditory procedures in one session in an IAC sound-treated room, including otoscopy, tympanometry, pure-tone threshold evaluation, auditory brainstem response per ear, and a three-channel auditory middle latency response in the right ear. The primary study outcomes were hearing thresholds measured $(\mathrm{dB} \mathrm{HL})$ at five frequencies, and evoked potential wave latencies (I, III, V, Na, Pa, Nb, Pb) and amplitudes (V-I, Na-Pa, Pa-Nb, Nb-Pb). It was hypothesized that SHS-exposed individuals would have poorer hearing sensitivity (threshold $>25 \mathrm{~dB} \mathrm{HL}$ ) and abnormal central auditory function (longer latencies; smaller amplitudes) based on evoked potentials. Statistical analyses focused on identification of group differences in hearing and central auditory function.

Results: All participants had normal hearing sensitivity (thresholds $\leq 25 \mathrm{~dB} \mathrm{HL}$ ) with no significant group differences. The V/I amplitude ratio in the right ear was significantly decreased in SHS-exposed individuals $(p<$ 0.05). Auditory brainstem response latencies were not significantly different between participant groups or ears. Wave $\mathrm{Pb}$ latency was significantly increased in SHS-exposed individuals $(p<0.01)$. Auditory middle latency response relative amplitudes were significantly different from each other at every electrode site $(\mathrm{Cz}, \mathrm{Fz}$, $\mathrm{C4}$ ) but not between groups. Overall, the $\mathrm{Na}-\mathrm{Pa}$ complex was highest in amplitude at all three electrode sites.

*Department of Speech Pathology and Audiology, University of South Alabama, Mobile, AL; †Honorary Research Fellow, School of Health Sciences, University of Kwazulu-Natal, South Africa; $\$$ Department of Biomedical Science, University of South Alabama, Mobile, AL

Corresponding author: Ishara Ramkissoon, Associate Professor of Audiology, University of South Alabama, Mobile, AL 36688; Email: ramkissoon@ southalabama.edu

The results of this study were presented at the 2014 HEAL Conference in Lake Como, Italy and at the 2015 ENT/SAAA/SASLHA Congress in Durban, South Africa. 
Conclusions: This preliminary study indicated toxic effects of SHS exposure by evoked potentials with decreased V/I amplitude ratio and longer (delayed) Pb latency in young adults. Further studies should corroborate these findings to facilitate clinical recommendations.

Key Words: ABR, AMLR, evoked potentials, secondhand smoke

Abbreviations: $A B R=$ auditory brainstem response; $A M L R=$ auditory middle latency response; $A N O V A=$ analysis of variance; EEG = electroencephalography; PTT = pure-tone thresholds; SD = standard deviations; SHS = secondhand smoke; SHS-E = exposed to secondhand smoke; SHS-U = unexposed to secondhand smoke

\section{INTRODUCTION}

$\mathrm{S}$ econdhand smoke (SHS) includes mainstream smoke exhaled by smokers and sidestream smoke emanating from lit cigarettes or tobacco products. Nonsmokers who are exposed to SHS are often labeled "passive smokers," and they potentially inhale many different toxic agents, including benzene, 1,3-butadiene, benzo[a]pyrene, and 4-(methylnitrosamino)-1-(3-pyridyl)1-butanone (World Health Organization International Agency for Research on Cancer, 2004). A Surgeon General Report indicated that 126 million nonsmokers are exposed to SHS in the United States (USDHHS, 2006). While active smoking and SHS exposure are different, they both can lead to similar negative health effects.

Many studies evaluated the impact of cigarette smoking and nicotine on the auditory system and indicated a strong association between smoking and prevalence of hearing loss in older adults (Cruickshanks et al, 1998). Acute amounts of nicotine administered to rats led to increased amplitude of wave $\mathrm{P} 20$ or $\mathrm{P} 50 / \mathrm{Pb}$ in humans (Metzger et al, 2007). In addition, young adult male smokers and male nonsmokers exposed to nicotine (patch) revealed increased wave $\mathrm{V}$ (auditory brainstem response [ABR]) amplitude (Harkrider et al, 2001; Gopal et al, 2009). Both younger and older smokers revealed an acute effect of nicotine based on increased $\mathrm{Na}-\mathrm{Pa}$ amplitude immediately after smoking a cigarette (Ramkissoon and Chambers, 2008). Young smokers had significantly higher $\mathrm{V}-\mathrm{Na}$ and $\mathrm{Nb}-\mathrm{Pb}$ relative amplitudes and shorter $\mathrm{Nb}$ latency in the auditory middle latency response (AMLR) (Ramkissoon and Beverly, 2014). Notwithstanding this evidence, the current authors did not assume that SHS exposure in nonsmokers resulted in the same auditory effects as cigarette smoking or nicotine.

A few investigations have evaluated the effects of SHS exposure on auditory functioning. SHS exposure is a risk factor for hearing loss, and was associated with increased upper respiratory tract and ear infections in infants and young children (Lyons, 1992; Jones et al, 2012). In aging adults (48-92 yr), nonsmokers who lived with a smoker were 1.95 times more likely to have a hearing loss than those not exposed to a household member who smoked (Cruickshanks et al, 1998). In nonsmoking adults (20$69 \mathrm{yr})$, SHS exposure was significantly associated with increased risk of hearing loss (Fabry et al, 2011). More specifically, never smokers had an increased risk (OR = 1.14) for low-frequency hearing loss and former smokers showed an increased risk for low-mid frequency (OR = $1.3)$ and high-frequency $(\mathrm{OR}=1.4)$ hearing loss. A retrospective study of adolescents aged 12-19 yr reported that those with SHS exposure were 1.83 times more likely to have sensorineural hearing loss (Lalwani et al, 2011). In the same study, higher serum cotinine levels were correlated with even greater hearing loss at higher frequencies with elevated pure-tone hearing thresholds measured at 2000,3000 , and $4000 \mathrm{~Hz}$ in SHS-exposed adolescents. Cotinine is a suitable biomarker of recent exposure to secondhand smoke, because of its half-life of $17 \mathrm{~h}$ (Etter et al, 2000). One animal study evaluated central nervous system (CNS) effects by assessing visual recognition memory and $\mathrm{ABR}$ in rhesus monkeys that were exposed to environmental tobacco smoke (Golub et al, 2007). Although visual recognition memory was decreased in monkeys exposed to SHS, there was no significant differences in the ABR between SHS-exposed and unexposed monkeys.

The rationale for the current project stems from limited current knowledge about the impact of SHS exposure on auditory function. Two previous studies used pure-tone audiometry to report a link between SHS exposure and hearing loss in adolescents and adults (Fabry et al, 2011; Lalwani et al, 2011). The only known study that evaluated SHS exposure using evoked potential methodology was conducted in animals (Golub et al, 2007). Little is known about the effect of SHS exposure on the human central auditory system, especially brainstem and cortical regions that contain nicotine receptors. In fact, electroencephalography (EEG) is a useful method to determine the physiological links of tobacco dependence (Panzano et al, 2010). Therefore, two evoked potential tests, ABR and AMLR, extracted from the EEG were proposed in the current project to examine the effects of SHS exposure, in postadolescent, young adult nonsmokers.

The specific research questions examined if there were any group differences in pure-tone hearing thresholds, ABR latencies, ABR amplitude, AMLR latencies, or AMLR amplitudes between individuals who reported SHS exposure and those who reported no SHS exposure. It was hypothesized that SHS-exposed individuals would demonstrate a decrease in pure-tone hearing 
sensitivity (poorer thresholds) and changes in central auditory system physiology reflected by increased amplitude and shorter latency of ABR and AMLR waves, a stimulant effect as shown by smoking, when compared to SHS-unexposed participants.

\section{METHODS}

\section{Study Design and Participants}

This was an age-matched, group design study conducted in the University of South Alabama campus. The goal for participant selection was to recruit nonsmokers who were exposed to SHS and had minimal noise exposure. Two hundred and eight prospective participants completed a questionnaire providing information about their medical, health, hearing, and smoking history. However, the majority of these individuals ( $\mathrm{n}=$ 180) were excluded from study participation because of reported tinnitus $(\mathrm{n}=54)$, current smoking or other tobacco use $(n=55)$, using medications that affect CNS function $(n=58)$, recent noise exposure $(n=40)$, neurological or mental disorders $(\mathrm{n}=3)$, or chemical exposure $(\mathrm{n}=19)$. Note that some excluded individuals had more than one of the exclusionary criteria listed above. Further, six individuals declined to participate further when invited. The 22 remaining self-reported nonsmokers with a history of being never smokers and in good overall health were included in the study. However, 5/22 individuals did report minimal noise exposure and 4/22 individuals reported occasional tinnitus; nevertheless, they were included because they met the most important criteria of being nonsmokers who were exposed to SHS. Participants signed an informed consent approved by the university IRB Office of Research Compliance. Two participants were rejected later because of (a) noisy ABR recordings with high artifact $(>10 \%)$ levels possibly because of unreported history of Bell's Palsy and (b) poor AMLR morphology and lack of replicable waveforms.

The 20 final participants were right-handed, nonsmokers with a mean age of 20 yr (range: 18-23), and they were grouped by their report of SHS exposure. The SHS-exposed (SHS-E) group ( $\mathrm{N}=10$; two males) reported exposure to SHS for an extensive period of time. All ten individuals reported a residential history of having lived in homes with one to two cigarette smokers. Also, nine of ten individuals reported 1-4 h daily SHS exposure from others smoking outside but nearby their homes. In addition, eight of ten of these individuals reported SHS exposure of $1-10 \mathrm{~h}$ in social settings, as recently as within the past $24 \mathrm{~h}$. All ten individuals indicated SHS exposure in work settings of less than $1 \mathrm{~h}$ daily. In contrast, nonsmokers who reported no exposure to secondhand smoke, and did not live or interact with any smokers were placed in the SHS-unexposed (SHS-U) group ( $\mathrm{N}=10$; three males). All participants in both groups denied use of any medications that affected CNS function.

Each participant provided a urine sample at a convenient time during the test session. An independent laboratory conducted biochemical analysis for cotinine levels using quantitative liquid chromatography- tandem mass spectrometry. Urinalysis verified participants $(\mathrm{N}=22)$ as nonsmokers. Threshold range for cotinine in urine was $0-4 \mu \mathrm{g} / \mathrm{L}$ for SHS-unexposed participants and 5-15 $\mu \mathrm{g} / \mathrm{L}$ for SHS-exposed participants based on a clinical range from Arup Laboratory, an independent facility. The cotinine levels confirmed self-reported nonexposure to SHS in all SHS-U participants, but only one individual in the SHS-E group had cotinine levels high enough to be classified as being SHS-exposed by biochemical analysis.

\section{Auditory Procedures}

All auditory procedures were conducted by the authors. Otoscopic examination conducted with a Welch Allyn otoscope (model 25020A) with a power handle (model 71050-C) and disposable specula confirmed unobstructed external auditory canals and good visualization of the tympanic membranes, bilaterally in each participant. Tympanometry conducted with a Madsen Zodiac 901 unit revealed bilateral normal middle ear pressure peak and compliance in all participants. Puretone audiometry and evoked potential tests were conducted inside a double-walled, sound-treated (Industrial Acoustics Company) booth using EARTONE 3A(Etymotic Research, Elk Grove, IL) insert earphones to deliver the auditory stimulus. A calibrated Grason-Stadler GSI-61 audiometer was used to measure hearing thresholds with a standard clinical procedure (modified HughsonWestlake) at five frequencies: 500, 1000, 2000, 3000, and $4000 \mathrm{~Hz}$, in each ear of every participant.

The ABR and AMLR evoked potential tests were performed with the Intelligent Hearing Systems Smart EP (version 5.10) system to evaluate the effects of SHS exposure in young adults for this first study. Testing time including setup was $35-40$ min per participant. SHS-U participants were scheduled at their convenience but SHS-E participants were scheduled within $24-48 \mathrm{~h}$ of their most recent SHS exposure. Silver-silver chloride $(\mathrm{Ag}-\mathrm{AgCl})$ disc type, reusable electrodes were placed on scalp sites (Cz, Fz, C4, M1, M2) according to the 10-20 International System (Jasper, 1958). Electrode impedance was maintained below $7 \mathrm{k} \Omega$, with interelectrode impedance balanced within 3-5 $\mathrm{k} \Omega$. If impedances were high, the respective scalp location was rescrubbed with Nuprep EEG gel before reapplication of electrodes. Participants sat in a recliner chair and kept their eyes open (awake) for the AMLR test. They were permitted to close their eyes and/or fall asleep for ABR testing. A minimum of two runs was required to confirm replication of the $A B R$ and AMLR waveforms before data analysis. However, 
Table 1. PTT per Ear in SHS-Unexposed and SHS-Exposed Participants

\begin{tabular}{|c|c|c|c|c|}
\hline \multirow[b]{3}{*}{ Frequency $(\mathrm{Hz})$} & \multicolumn{4}{|c|}{ Mean (SD), dB HL } \\
\hline & \multicolumn{2}{|c|}{ SHS Unexposed } & \multicolumn{2}{|c|}{ SHS Exposed } \\
\hline & Left Ear & Right Ear & Left Ear & Right Ear \\
\hline 500 & $4.5(6.8)$ & $5.5(6.4)$ & $7.0(4.2)$ & $9(4.5)$ \\
\hline 1000 & $4.5(6.8)$ & $5.5(6.8)$ & $7.0(5.8)$ & $7(7.1)$ \\
\hline 2000 & $3.0(7.1)$ & $6.0(6.9)$ & $4.0(7.3)$ & $6.5(4.7)$ \\
\hline 3000 & $5.0(6.6)$ & $8.0(7)$ & $3.0(8.8)$ & $3.0(6)$ \\
\hline 4000 & $4.0(5.1)$ & $2.0(6.7)$ & $2.0(6.7)$ & $2.0(6.7)$ \\
\hline
\end{tabular}

Note: $\mathrm{n}=10$ per participant group.

majority (70-80\%) of participants required a third run to confirm replication of the respective waveform.

The ABR test was acquired with a $70 \mathrm{~dB}$ nHL (intensity) click stimulus, $100 \mu$ sec in duration, presented monaurally at a rate of 17.1 clicks per second to each ear. Responses between the noninverting $(\mathrm{Cz})$ and inverting (M1 or M2) electrodes were recorded through an amplifier set to bandpass activity from 10 to $3000 \mathrm{~Hz}$. A total of 1,024 stimulus sweeps were averaged per ear in a $12-\mathrm{ms}$ time window. The AMLR was acquired with a $70 \mathrm{~dB}$ nHL click stimulus, presented monaurally at a rate of 8.1 clicks per second to the right ear. AMLR responses were obtained in a three-channel recording between the noninverting $(\mathrm{Fz}, \mathrm{Cz}, \mathrm{C} 4)$ and inverting linked (M1/M2) electrodes with an amplifier set to bandpass activity from 10 to $200 \mathrm{~Hz}$. A total of 512 stimulus sweeps were averaged within a $102-\mathrm{ms}$ time window.

\section{Data and Statistical Analysis}

Audiometric data from each participant yielded the dependent variables, including pure-tone thresholds (PTT) at five frequencies per ear, ABR latencies (I, III, V) and V-I amplitude ratio per ear, and AMLR latencies and relative amplitudes from the right ear. Of the two to three evoked potential waveforms acquired per ear (ABR) and electrode (AMLR) in each participant, the two runs that replicated best were averaged using a sweep-weighted function to yield a single waveform for analysis of latency and amplitude. Thus, five evoked potential waveforms (AMLR-Cz, AMLR-C4,

Table 2. Mean ABR Latencies (msec) and Amplitude $(\mu \mathrm{V})$ per Ear in SHS-Unexposed and SHS-Exposed

Participants

\begin{tabular}{|c|c|c|c|c|}
\hline \multirow[b]{2}{*}{ Component } & \multicolumn{2}{|c|}{ SHS Unexposed } & \multicolumn{2}{|c|}{ SHS Exposed } \\
\hline & Left Ear & Right Ear & Left Ear & Right Ear \\
\hline I & $1.70(0.12)$ & $1.78(0.11)$ & $1.70(0.12)$ & $1.79(0.13)$ \\
\hline III & $3.81(0.39)$ & $3.87(0.22)$ & $3.96(0.21)$ & $3.96(0.23)$ \\
\hline V & $5.59(0.28)$ & $5.58(0.30)$ & $5.85(0.36)$ & $5.81(0.41)$ \\
\hline $\begin{array}{l}\text { V/I amplitude } \\
\text { ratio }\end{array}$ & $1.75(0.94)$ & $3.26(2.01)$ & $1.66(1.02)$ & $1.65(1.05)$ \\
\hline
\end{tabular}

Note: SDs are in parentheses, $\mathrm{n}=20$.
AMLR-Fz, ABR-right, and ABR-left) were analyzed per participant by a trained graduate student who was blinded to participant grouping. Absolute latencies (I, III, V, $\mathrm{Na}, \mathrm{Pa}, \mathrm{Nb}, \mathrm{Pb}$ ) and relative amplitudes (V-I, $\mathrm{Na}-\mathrm{Pa}, \mathrm{Pa}-\mathrm{Nb}, \mathrm{Nb}-\mathrm{Pb}$ ) were determined for each waveform with IHS software, version 3.95.

Twenty percent of waveforms were reanalyzed by a second trained student for reliability analysis. Agreement between the first and second rater was based on an acceptable range of latency and amplitude measures, namely within $0.2 \mathrm{~ms}$ for $\mathrm{ABR}$ waves I, III, and V; within $3.0 \mathrm{~ms}$ for AMLR waves $\mathrm{Na}, \mathrm{Pa}, \mathrm{Nb}$, and $\mathrm{Pb}$; and within $0.3 \mu \mathrm{V}$ for AMLR relative amplitudes $\mathrm{Na}-\mathrm{Pa}, \mathrm{Pa}-\mathrm{Nb}$, and $\mathrm{Nb}-\mathrm{Pb}$. Inter-rater reliability using the above criteria was $95 \%$ for $\mathrm{ABR}$ latency, $75 \%$ for $\mathrm{ABR}$ amplitude, 91\% for AMLR latency, and 92\% for AMLR amplitude.

The 39 data points per participant were entered into a spreadsheet to calculate means and standard deviations (SDs). Statistical analysis with SPSS, version 20 began with omnibus analysis of variance (ANOVA) to evaluate any differences in PTT, ABR latencies, AMLR latencies, ABR amplitude, and AMLR relative amplitudes between two participant groups (SHS-E, SHS-U) with $p<0.05$ set to determine significance. Follow-up univariate ANOVA and paired samples $T$-Tests were conducted as needed.

\section{RESULTS}

$\mathrm{E}$ ach participant had normal otoscopic examination and tympanometry indicated by a compliance peak of $0.3 \mathrm{~mL}$ or greater, in each ear. Statistical analyses were conducted using the preplanned grouping of participants into SHS-E and SHS-U groups based on selfreported SHS exposure.

\section{PTT}

All participants in both groups had normal hearing sensitivity, defined by PTT $\leq 25 \mathrm{~dB}$ HL. Specifically, individual hearing thresholds ranged from -10 to $15 \mathrm{~dB}$ HL at each frequency tested. Group mean thresholds and SDs per frequency and ear are shown in Table 1. To determine whether PTT differed between participant groups, a $2 \times 5 \times 2$ repeated measures ANOVA was conducted with two within-subjects factors: Ear (Right, Left), and PTT (500, $1000,2000,3000,4000 \mathrm{~Hz}$ ), and one between-subjects factor, Group (Exposed, Unexposed). There was a significant interaction of PTT by Group, $F_{(4,72)}=2.76, p<0.05$ with a power of 0.73 and a small effect size $\left(\eta_{p}^{2}=0.13\right)$; this finding was because the SHS-E group having slightly poorer low-frequency $(500,1000 \mathrm{~Hz})$ thresholds than the SHS-U group (Table 1). There was also a significant main effect of PTT, $F_{(4,72)}=4.55, p<0.01$ with a power of 0.93 and a small effect size $\left(\eta_{p}^{2}=0.20\right)$ due to subtle differences in threshold among the frequencies evaluated (Table 1). As there were no effects involving Ear, the PTT were collapsed 


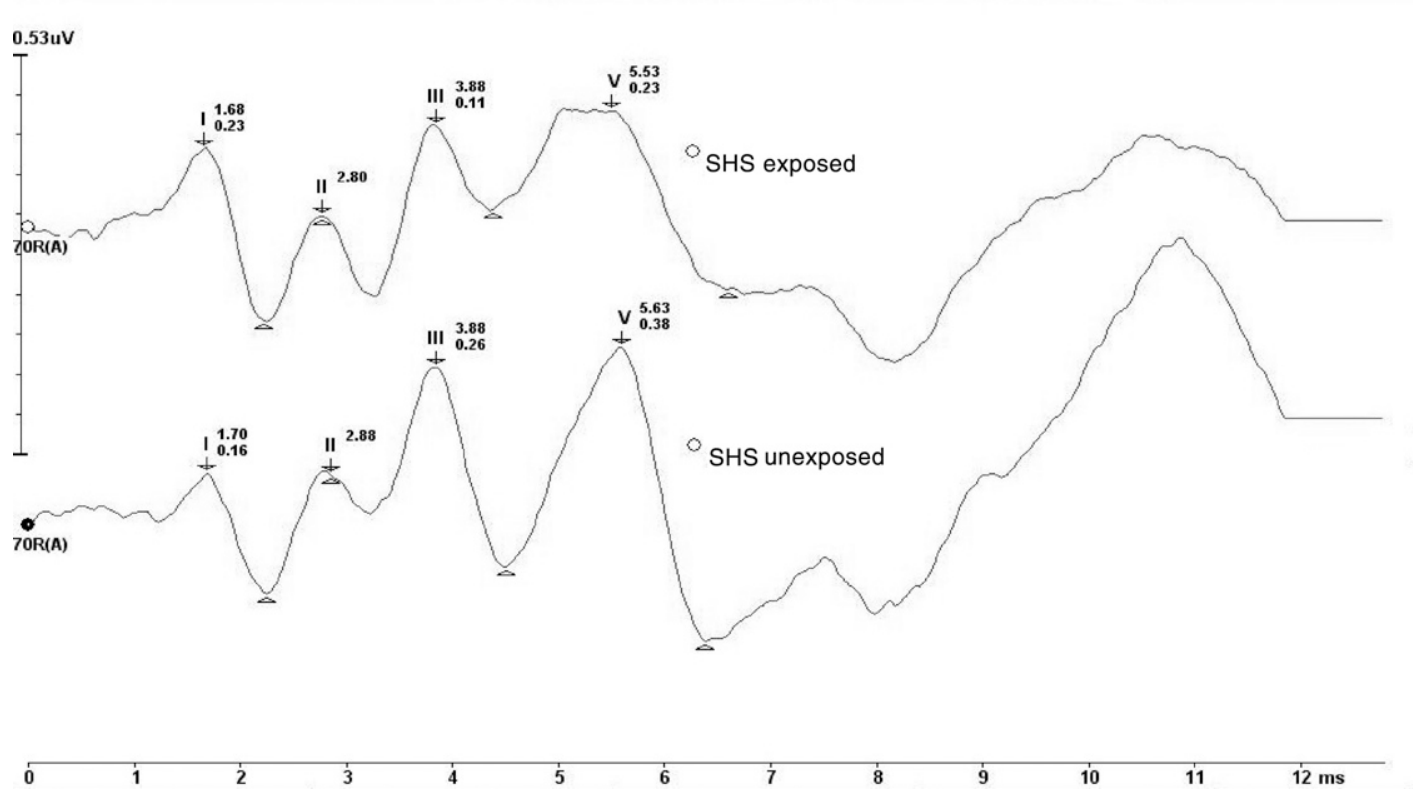

Figure 1. Grand average ABR waveform from the right ear of SHS-exposed (upper figure) and unexposed (lower figure) participants, $\mathrm{n}=10$ per group.

across the right and left ears for univariate analyses of each PTT, with Group as the between-subjects factor. For each PTT analyzed, there were no significant group differences; the power was very low for these analyses and together with the small effect sizes, suggests a spurious finding.

\section{ABR}

The ABR was recorded from the right and left ear separately in each participant. The main ABR waves, labeled I, III, and V were consistently recorded in both ears. Mean ABR latencies and V/I amplitude ratio with SDs are shown in Table 2.

To determine whether ABR wave latencies were significantly different between participant groups, a threefactor repeated measures ANOVA was conducted with two within-subjects factors: Ear (Right, Left), and Component (I, III, V), and one between-subjects factor, Group (Exposed, Unexposed). There was a significant main effect of Component, $F_{(2,36)}=2,828.14, p<0.001$ with a power of
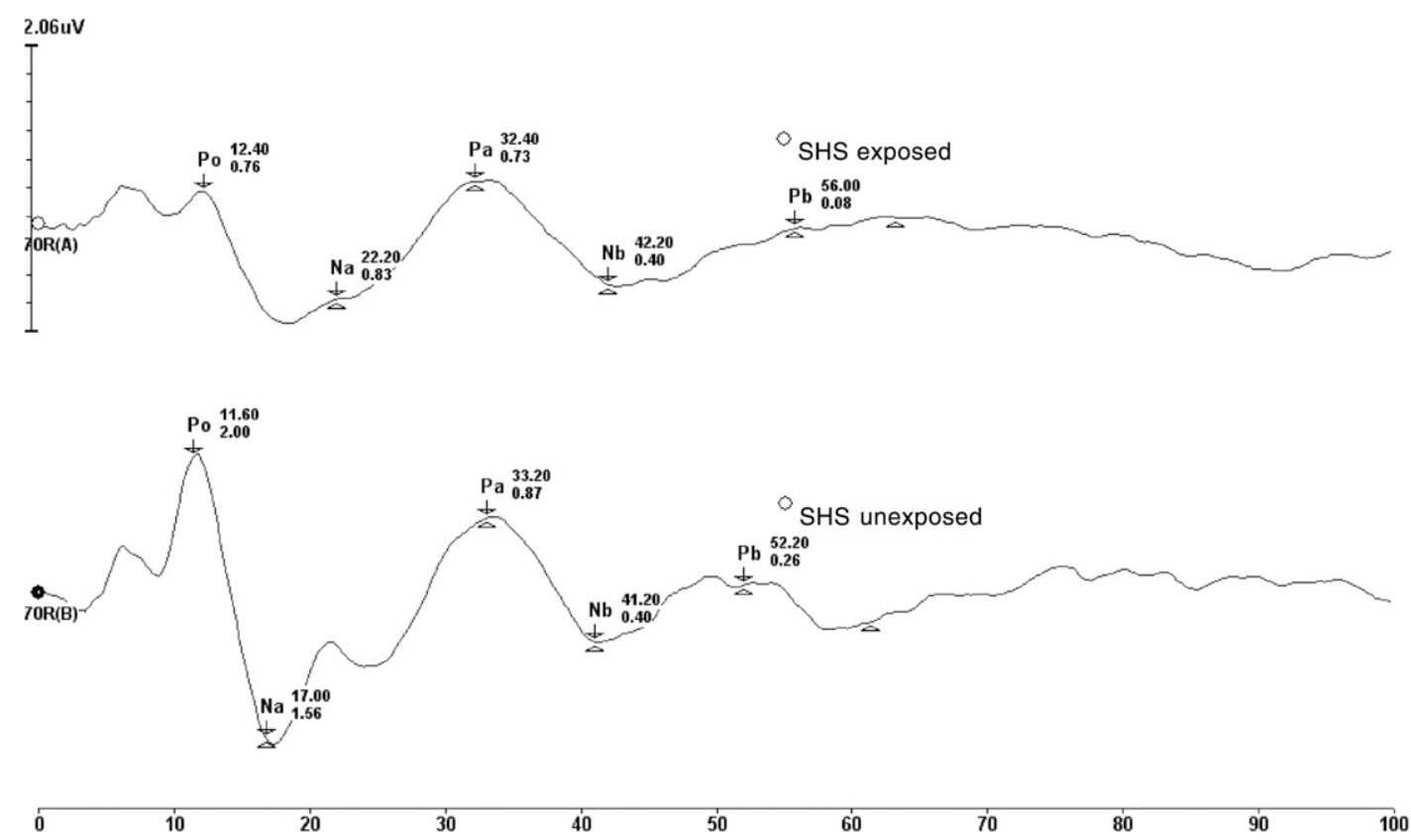

40

50

60

70

$80 \quad 90 \quad 100$ r

Figure 2. Grand average AMLR waveform from Fz electrode in SHS-exposed (upper figure) and unexposed (lower figure) participants, $\mathrm{n}=10$ per group. 


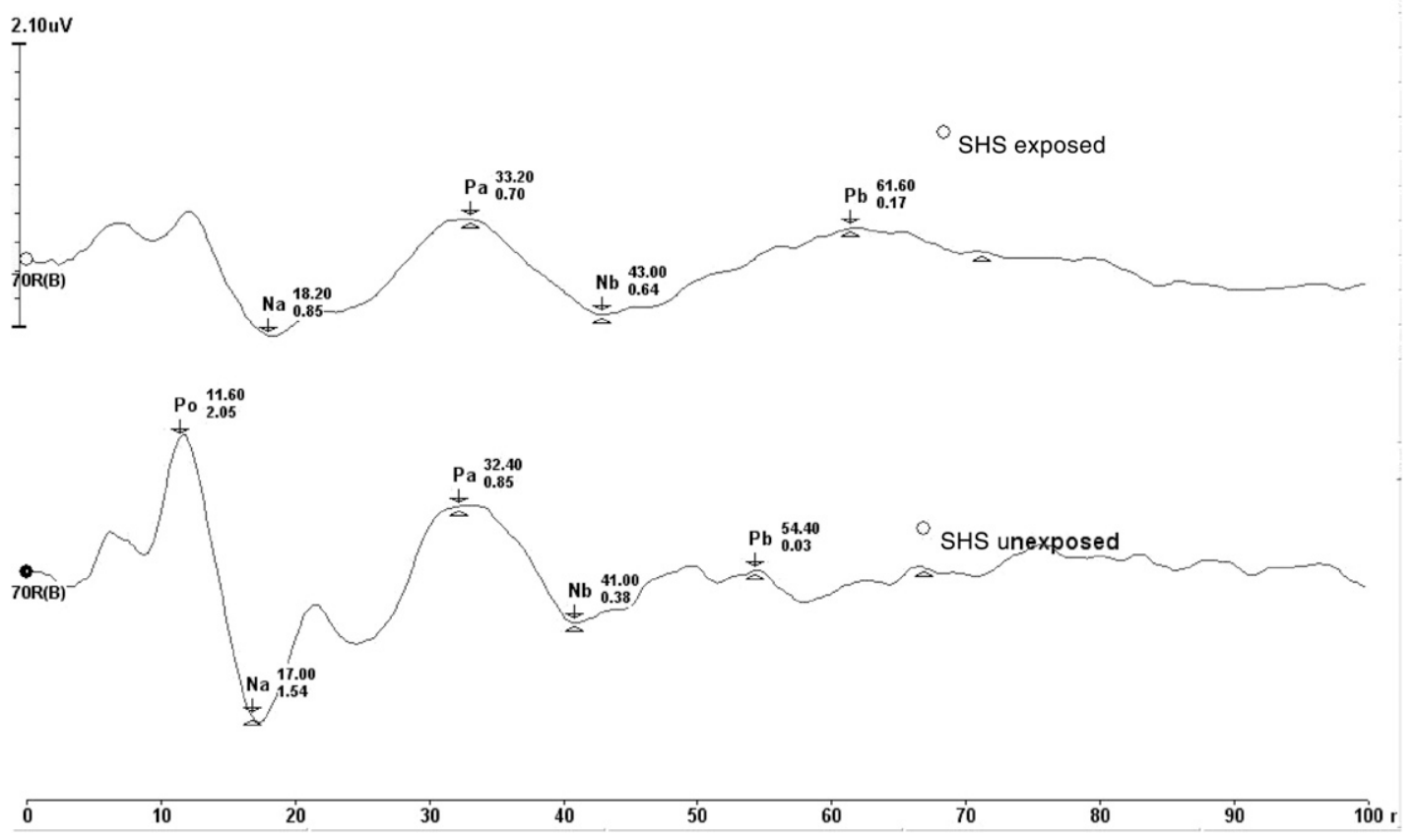

Figure 3. Grand average AMLR waveform from $\mathrm{Cz}$ electrode in SHS-exposed (upper figure) and unexposed (lower figure) participants, $\mathrm{n}=10$ per group.

1.0 and a large effect size $\left(\eta_{p}^{2}=0.99\right)$ due to the latency of waves I, III, and V being different from each other. There were no significant differences involving Group or Ear. The main effect of Component did not warrant follow-up testing because it is an expected finding for these evoked potential waves to differ systematically in latency by virtue of their neural generator location in the auditory system.

To determine whether the ABR V/I amplitude ratio was significantly different between groups, a two-factor $(2 \times 2)$ ANOVA was conducted with Ear (Right, Left) as withinsubjects and Group (Exposed, Unexposed) as betweensubjects factors. There was a significant interaction of Ear by Group, $F_{(1,18)}=5.14, p<0.05$ with a power of 0.57 and a moderate effect size $\left(\eta_{p}^{2}=0.22\right)$, and a significant main effect of Ear, $F_{(2,36)}=5.05, p<0.05$ with a power of 0.56 and a moderate effect size $\left(\eta_{p}^{2}=0.21\right)$ There was no main effect of Group. To determine the source of the interaction, univariate ANOVA conducted per Ear revealed a significant group difference in the $\mathrm{V} / \mathrm{I}$ amplitude ratio for the right ear, $F_{(1,18)}=$ 4.97, $p<0.05$ with a power of 0.56 and a moderate effect size $\left(\eta_{p}^{2}=0.22\right)$, but not the left ear. This was because of SHS-exposed participants having a lower mean V/I amplitude ratio (1.65), compared with nonexposed (3.25) individuals, in the right ear. The right ear grand average ABR waveforms per participant group are shown in Figure 1.

\section{AMLR}

AMLR from the right ear of each participant was consistently recorded with waves $\mathrm{Na}, \mathrm{Pa}, \mathrm{Nb}$, and $\mathrm{Pb}$ observed

Table 3. AMLR Latencies and Amplitudes from Three Electrode Sites with Right Ear Stimulus in SHS-Unexposed and SHS-Exposed Participants

\begin{tabular}{|c|c|c|c|c|c|c|}
\hline \multirow[b]{3}{*}{ Component } & \multicolumn{6}{|c|}{ Mean Latency in msec (SD) } \\
\hline & \multicolumn{3}{|c|}{ SHS Unexposed } & \multicolumn{3}{|c|}{ SHS Exposed } \\
\hline & $\mathrm{Fz}$ & $\mathrm{Cz}$ & $\mathrm{C} 4$ & $\mathrm{Fz}$ & $\mathrm{Cz}$ & $\mathrm{C} 4$ \\
\hline$\overline{\mathrm{Na}}$ & $19.6(3.1)$ & $19.9(3.0)$ & $19.1(2.8)$ & $19.6(2.3)$ & $18.7(2.2)$ & $18.4(2.2)$ \\
\hline $\mathrm{Pa}$ & $32.8(2.4)$ & $33.0(2.4)$ & $32.4(2.5)$ & $32.8(2.1)$ & $32.5(2.1)$ & $32.5(2.3)$ \\
\hline $\mathrm{Nb}$ & $43.0(4.2)$ & $42.6(3.7)$ & $42.4(3.0)$ & $45.4(2.3)$ & $45.2(2.2)$ & $43.8(2.2)$ \\
\hline \multirow[t]{2}{*}{$\mathrm{Pb}$} & $53.3(3.8)$ & $53.2(3.7)$ & $53.7(2.9)$ & $56.2(3.6)$ & $58.4(3.0)$ & $56.8(3.2)$ \\
\hline & \multicolumn{6}{|c|}{ Mean Amplitude in $\mu \mathrm{V}$ (SD) } \\
\hline$\overline{\mathrm{Na}-\mathrm{Pa}}$ & $1.9(1.9)$ & $2.1(1.8)$ & $1.9(1.8)$ & $1.3(0.6)$ & $1.1(0.7)$ & $1.3(0.6)$ \\
\hline $\mathrm{Pa}-\mathrm{Nb}$ & $1.2(0.9)$ & $1.4(1.0)$ & $1.3(0.8)$ & $1.0(0.5)$ & $0.9(0.5)$ & $0.9(0.5)$ \\
\hline $\mathrm{Nb}-\mathrm{Pb}$ & $0.7(0.6)$ & $0.8(0.6)$ & $0.8(0.6)$ & $0.6(0.6)$ & $0.9(0.5)$ & $0.7(0.6)$ \\
\hline
\end{tabular}

Note: SDs are in parentheses, $\mathrm{n}=10$. 
at all three electrode sites. Postauricular muscle reflex was noted in the AMLR waveform in five of ten unexposed participants and three of ten exposed participants. Grand average AMLR waveforms comparing two participant groups were created per electrode site, for example, see Figure 2 for $\mathrm{Fz}$ and Figure 3 for $\mathrm{Cz}$ waveforms. Mean AMLR latencies and relative amplitudes with SDs are shown in Table 3.

\section{AMLR Latencies}

To examine whether AMLR wave latencies were significantly different between the SHS exposed and unexposed groups, a three-factor $(3 \times 4 \times 2)$ repeated measures ANOVA was conducted with two withinsubjects factors: Electrode $(\mathrm{Cz}, \mathrm{Fz}, \mathrm{C} 4)$, Component $(\mathrm{Na}, \mathrm{Pa}, \mathrm{Nb}, \mathrm{Pb})$, and one between-subjects factor, Group (Exposed, Unexposed). Mauchly's test of sphericity was not significant, so sphericity is assumed. There was a significant interaction of Component with Group, $F_{(3,54)}=5.24, p<0.01$ with a power of 0.91 and a moderate effect size $\left(\eta_{p}^{2}=0.226\right)$ due to the SHS-E group having longer latencies for some wave components. There was also a significant main effect of Component, $F_{(3,54)}=1,037.66, p<0.01$ with a power of 1.0 and a large effect size $\left(\eta_{p}^{2}=0.983\right)$ due to the latency of waves $\mathrm{Na}, \mathrm{Pa}, \mathrm{Nb}$, and $\mathrm{Pb}$ being different from each other. No follow-up testing was done for the Component main effect, as it is a known and expected finding for AMLR waves to have different latencies because of their neural generator location in the auditory system. There were no significant main effects of Group or Electrode.

The significant Latency by Group interaction was followed up with univariate analyses conducted per Component variable, collapsed across electrode because there were no significant differences among electrodes. These tests revealed no significant Group differences for mean latencies of waves $\mathrm{Na}, \mathrm{Pa}$, and $\mathrm{Nb}$. However, there was a significant group difference for mean $\mathrm{Pb}$ latency, $F_{(1,18)}=$ $11.37, p<0.01$ with a power of 0.89 and a small effect size $\left(\eta_{p}^{2}=0.39\right)$. This was due to SHS-exposed individuals having longer mean $\mathrm{Pb}$ latency (57.15 ms) compared with SHS-unexposed (53.06 ms) individuals.

\section{AMLR Amplitudes}

Mean AMLR relative amplitudes with SDs are shown in Table 3. To determine whether AMLR relative amplitudes were significantly different between groups, a three-factor $(3 \times 3 \times 2)$ repeated measures ANOVA was conducted with two within-subjects factors, Electrode (Cz, Fz, C4), Component (Na-Pa, $\mathrm{Pa}-\mathrm{Nb}$, $\mathrm{Nb}-\mathrm{Pb}$ ), and one between-subjects factor, Group (Exposed, Unexposed). As Mauchly's test of sphericity revealed significance, sphericity cannot be assumed and Greenhouse-Geisser results are reported. There was a significant interaction of Electrode by Component by Group, $F_{(4,72)}=3.53, p<0.05$ with a power of 0.69 and a small effect size $\left(\eta_{p}^{2}=0.16\right)$. This significant interaction of Electrode by Component by Group was followed up with repeated measures $3 \times 2$ (electrode $\times$ group) ANOVAs per Component variable. There were no significant main effects or interactions for these three follow-up analyses because of similar relative amplitude measurements obtained per electrode in both groups; the power was very low for these analyses, suggesting this was a spurious finding.

The omnibus ANOVA for analyzing AMLR amplitude also revealed a significant main effect of Component, $F_{(2,36)}=11.07, p<0.01$ with a power of 0.92 and an small effect size $\left(\eta_{p}^{2}=0.38\right)$ due to relative amplitudes being different from each other. This main effect of Component was followed up with repeated measures $3 \times 2$ ANOVAs per electrode site. For site $\mathrm{Cz}$, there was significant main effect of Component, $F_{(2,36)}=$ $7.42, p<0.05$ with a power of 0.77 , and a moderate effect size $\left(\eta_{p}^{2}=0.29\right)$. Paired samples $T$-Tests for the three components at the $\mathrm{Cz}$ electrode site revealed significance $(p<0.05)$ on all three relative amplitude components that were compared: $\mathrm{Na}-\mathrm{Pa}(1.56)$ was different than $\mathrm{Pa}-\mathrm{Nb}$ (1.14), $\mathrm{Na}-\mathrm{Pa}$ (1.56) was different than $\mathrm{Nb}-\mathrm{Pb}(0.86)$, and $\mathrm{Pa}-\mathrm{Nb}$ (1.14) was different than $\mathrm{Nb}-\mathrm{Pb}$ (0.86). At the $\mathrm{Fz}$ electrode site, there was a significant main effect of Component, $F_{(2,36)}=11.50, p<$ 0.01 with a power of 0.93 , and a large effect size $\left(\eta_{p}^{2}=\right.$ 0.39 ). Paired samples $T$-Tests for the three components at $\mathrm{Fz}$ revealed significance $(p<0.01)$ on all three relative amplitude components that were compared: $\mathrm{Na}-\mathrm{Pa}$ (1.58) was different than $\mathrm{Pa}-\mathrm{Nb}$ (1.12), Na-Pa (1.58) was different than $\mathrm{Nb}-\mathrm{Pb}(0.68)$, and $\mathrm{Pa}-\mathrm{Nb}$ (1.12) was different than $\mathrm{Nb}-\mathrm{Pb}$ (0.68). At electrode site $\mathrm{C} 4$, there was a significant main effect of Component, $F_{(2,36)}=13.06, p<0.01$ with a power of 0.95 , and a large effect size $\left(\eta_{p}^{2}=0.42\right)$. Paired samples $T$-Tests for the three components at $\mathrm{C} 4$ revealed significance $(p<0.01)$ on all three relative amplitude components that were compared: $\mathrm{Na}-\mathrm{Pa}$ (1.64) was different than $\mathrm{Pa}-\mathrm{Nb}$ (1.13), $\mathrm{Na}-\mathrm{Pa}$ (1.64) was different than $\mathrm{Nb}-\mathrm{Pb}(0.74)$, and $\mathrm{Pa}-\mathrm{Nb}$ (1.13) was different than $\mathrm{Nb}-\mathrm{Pb}(0.74)$. Note that $\mathrm{Na}-\mathrm{Pa}$ amplitude was largest in amplitude overall at all three electrode sites. The grand average AMLR waveforms per participant group for electrode $\mathrm{Fz}$ and $\mathrm{Cz}$ are shown in Figures 2 and 3, respectively.

\section{DISCUSSION}

The current study sought to examine hearing 1 thresholds and central auditory function through pure-tone audiometry, ABR, and AMLR in young adult nonsmokers who reported being exposed to SHS compared with an age- and gender-matched group who 
reported no SHS exposure. It was anticipated that SHS exposure might impair hearing as shown in adolescents (Lalwani et al, 2011) and yield a stimulant effect similar to that shown by nicotine patch administration in young adult nonsmokers (Harkrider et al, 2001).

\section{Hearing Thresholds}

The first hypothesis in the current study predicted that young adult nonsmokers who reported exposure to SHS would reveal poorer hearing thresholds than those unexposed to SHS. This hypothesis was not supported because pure-tone audiometry results, combined from both ears revealed no significant group differences for hearing thresholds at the five frequencies (500, $1000,2000,3000,4000 \mathrm{~Hz}$ ) evaluated. Previously, adolescents (12-19 yr) exposed to passive smoking showed poorer hearing thresholds in the higher frequencies, 2000, 3000, and $4000 \mathrm{~Hz}$ (Lalwani et al, 2011), but our SHS-E group did not demonstrate such differences in hearing thresholds. It is noteworthy, however, that the statistically significant group difference reported previously (Lalwani et al, 2011) was based on pure-tone threshold differences of 1-3 dB, which has minimal clinical significance. Furthermore, both exposed and nonexposed adolescents in the study by Lalwani et al had normal hearing with thresholds $<10 \mathrm{~dB}$ HL which is just like the young adults in the current study, that is, there was no clinically significant hearing impairment reported. Methodological differences between the current and the previous (Lalwani et al, 2011) study may have also contributed to the discrepant results. Specifically, the previous report retrospectively examined population-based data from adolescents who had been living in homes with smokers whereas the current study participants were young adults living in college campus residence halls, many of which were smoke-free environments. Thus, their reports of living in SHS environments related to their permanent address (parents' homes), not their actual current living places.

The current participants with zero smoking history but reported SHS exposure did not reveal any robust low-frequency hearing loss as was reported for never smokers (Fabry et al, 2011); however, those never smokers included aging adults up to $69 \mathrm{yr}$ who might have had other medical risks associated with developing hearing loss. In contrast, the current group of nonsmokers were young, healthy adults aged $18-23$ yr with no medicine use, minimal tinnitus or noise exposure, and they reported only SHS exposure as a variable of consideration for hearing loss. It is interesting, however, that the current omnibus analysis for pure-tone data revealed slightly poorer low-frequency $(500,1000 \mathrm{~Hz})$ thresholds in SHS-exposed participants. This current result had a small effect size, and subsequent follow-up analysis indicated it was a spurious finding. Perhaps future studies with a larger sample size might be able to tease out any significant impact on low-frequency pure-tone hearing thresholds.

\section{Central Auditory Function}

Two evoked potential tests (ABR, AMLR) conducted in this study facilitated an in-depth neurologic functional assessment of the central auditory nervous system in young adult nonsmokers who reported being exposed to secondhand smoke. The data were compared with a group of age and gender-matched individuals who had no reported SHS exposure. Statistical analyses thus focused on identifying group differences. Analysis of the ABR waveforms revealed an interesting finding in the amplitude measurement. For ABR evaluation, the V/I ratio indicates brainstem neurologic function (Hall, 2007) by comparing the voltage or size (amplitude) of wave $V$ to wave I. In normal hearing individuals with intact neurologic systems, wave $\mathrm{V}$ is typically twice as large as wave I, thus a normal V/I ratio should be 0.5 or higher. In the current study, both participant groups revealed a mean V/I ratio within normal range but group differences were evident. Specifically, the V/I amplitude ratio was significantly different between participant groups, for the right ear only. This was because of SHS-exposed individuals having a lower mean V/I amplitude ratio (1.65) compared with the nonexposed (3.25) individuals. This lower amplitude ratio observed in SHS-exposed individuals supports a toxicity effect, that is, decreased amplitude observed in the current study among nonsmokers with reported SHS exposure is contrary to the stimulant effect seen in smokers. The current finding implies that SHS exposure led to poorer evoked potentials because the magnitude of the neurologic response was smaller in exposed individuals. Reduced wave amplitude reflects lower measured voltage and could indicate possible reduction in synchronous neuronal firing, an important requirement for ABR acquisition. Another possibility is that SHS-exposure altered the responses of low-characteristic frequency neurons in the brainstem that primarily contribute to wave $\mathrm{V}$ amplitude, and similarly impacted cochlear neurons which could explain the slight differences in low-frequency PTT observed in the current group of SHS-exposed individuals. Together, these two findings further support a toxicity effect of SHS that likely reduced low-characteristic frequency neural activity. Overall, the current finding of reduced V/I amplitude ratio in SHS-exposed individuals does not support our SHS stimulant hypothesis that was based on previous research reporting that cigarette smoking led to excitation of nicotine receptors in the CNS (Harkrider et al, 2001; Gopal et al, 2009). There were also methodological differences between the current study 
and previous investigations, including measurement parameters (stimulus intensity level; wave $\mathrm{V}$ versus V/I ratio), participant gender (males only versus primarily female), and classification of smoke exposure (smokers versus nonsmokers).

In contrast to amplitude, the ABR latency analyses revealed no significant group differences; thus not supporting our hypothesis for a stimulant effect of SHS. Although there was a significant main effect of Component within each group of participants, follow-up statistical analyses for these differences in component latency were not performed because of existing research and clinical knowledge. Specifically, it is known and expected for ABR waves I, III, and V to be systematically different in latency or time of occurrence after auditory stimulation. There are no known previous studies that have addressed ABR latency differences due to SHS exposure.

Analyses of the AMLR waveforms acquired from each participant revealed some interesting latency differences between the participant groups. It was hypothesized that AMLR latencies would be decreased in individuals exposed to SHS. In contrast, the current results revealed that wave $\mathrm{Pb}$ latency was increased, that is $\mathrm{Pb}$ latency was significantly longer in SHS-exposed $(57.15 \mathrm{~ms})$ compared with unexposed $(53.06 \mathrm{~ms})$ participants. This finding does not support the stimulant hypothesis for effects of SHS exposure as observed in rats after administration of acute nicotine (Metzger et al, 2007). Instead, the longer AMLR latency observed in individuals exposed to SHS suggests a toxicity effect because wave $\mathrm{Pb}$ was delayed in SHS-exposed individuals, that is it occurred later in time after auditory stimulus presentation when compared with waveforms from individuals who were unexposed to SHS. A possible explanation for this longer latency could relate to impaired neurologic functioning of nicotinic receptors and auditory neurons that are usually excitatory in the CNS projection pathways. It is noteworthy that this delayed latency was unique to the AMLR, as it did not occur in the ABR. It is possible that the AMLR is more sensitive to cotinine (byproduct of nicotine) effects from SHS exposure than the ABR because of the overlapping anatomical structures involved in AMLR generation and nicotine uptake in the CNS. Specifically, whereas AMLR neural generators include the primary auditory and association cortices (Moller, 1994), midbrain (Hashimoto, 1982), reticular formation, and auditory thalamocortical pathways (Kraus et al, 1994), specific nicotine-binding sites are located in some of the same structures including the reticular formation, midbrain, and thalamus (Le Houezec and Benowitz, 1991). In fact, the reticular formation which contributes to AMLR generation also modulates the state of arousal (Bear et al, 2001) as does nicotine.

With respect to AMLR amplitude, it was hypothesized that SHS-exposed individuals would have larger relative amplitudes than unexposed participants but the current findings did not reveal any group differences. There was, however, significantly higher amplitudes for the earlier AMLR waves $(\mathrm{Na}-\mathrm{Pa})$ than later waves $(\mathrm{Nb}-\mathrm{Pb})$, across all three electrode sites and in both participant groups. Specifically, $\mathrm{Na}-\mathrm{Pa}$ amplitude was 1.5-2 times larger than $\mathrm{Nb}-\mathrm{Pb}$ amplitude at each electrode site. However, this finding is not surprising as the current test protocol parameters were set to optimize acquisition of the earlier AMLR waves; it requires a much slower stimulus repetition rate and consequently longer test acquisition time to record the later waves, especially $\mathrm{Pb}$. As a first study exploring SHS effects in auditory evoked potentials, we focused on acquisition of early AMLR waves. Nevertheless, the current finding does indicate that secondhand smoke seems to have affected earlier AMLR waves differently than the later waves, similar to the differential impact of cigarette smoking on the AMLR reported previously (Ramkissoon and Beverly, 2014). No other studies are known to have addressed this question for SHS-exposed individuals. Taken together, the current evoked potential findings of decreased ABR amplitude (V/I ratio) and increased AMLR latency (wave $\mathrm{Pb}$ ) support the suggestion that indirect smoke exposure impacts the auditory system differently (toxicity effect) than smoking or direct nicotine administration (stimulant effect).

\section{Limitations}

Participant age and living environment may have influenced our findings. The data analyzed in this study were obtained from young adults who reported having long-term exposure to SHS. However, the majority of them were not living consistently in homes with smokers as they resided on a smoke-free college campus. Thus, their SHS exposure at home was inconsistent. In addition, some of the current participants reported SHS exposure duration as low as one hour daily from smokers nearby their homes which might have led to barely detectable cotinine levels as discussed below.

For the purpose of this study, participant grouping was based on self-reported SHS exposure. Urine analysis was selected to measure cotinine levels as a biomarker of SHS exposure, and supplemented participants' self-report. Although all SHS-U participants were confirmed to be unexposed by cotinine levels of $<5 \mu \mathrm{g} / \mathrm{L}$, only one SHS-E participant was categorized as SHS-exposed with a cotinine level of $10 \mu \mathrm{g} / \mathrm{L}$. Ideally, all 10 individuals who reported being SHS exposed would have revealed cotinine levels to support the self-report but even infants with known passive smoking did not always show positive cotinine results in urine (Lyons, 1992). In addition, 
current participants were never smokers and therefore were not likely to show barely detectable cotinine levels; this is different than the adolescents in the Lalwani et al (2011) study who were classified as SHS-exposed even though they might have smoked as recently as six days before their cotinine levels were assessed. Furthermore, recent reports indicated that methods to accurately determine SHS are still unclear but urine tests remain a popular, noninvasive method to corroborate with self-report to determine SHS exposure (Avila-Tang et al, 2013).

The current study did not gather socioeconomic background information from participants which might possibly influence some of the differences found between SHS-exposed and unexposed individuals. This factor should be given consideration in future studies. Also, the current study focused on hearing and central auditory function specifically. However, peripheral effects of SHS exposure have yet to be explored, and including tests like otoacoustic emissions and extended high-frequency puretone audiometry in a future investigation would potentially yield new and valuable information.

\section{CONCLUSION}

$\mathrm{T}$ o our knowledge, this is the first study to evaluate the effects of SHS exposure on central auditory function in young adults. Key findings indicated a possible toxicity effect of SHS because AMLR wave $\mathrm{Pb}$ latency was delayed, and $A B R$ V/I amplitude ratio was reduced in the right ear of SHS-exposed individuals. Pure-tone audiometry did not reveal any significant differences in hearing thresholds between exposed and unexposed young adults; however, slight differences in low-frequency thresholds were observed in this sample. Further investigation is needed to confirm these findings, and to evaluate the impact of SHS on central auditory functioning in individuals who are consistently exposed to SHS, such as teenagers living in homes with smokers. Future research might also aim to quantify SHS exposure in public and private spaces to address potential environmental health concerns given these early indications of possible toxicity effects on central auditory pathway functions.

Acknowledgments. This research project was partially supported by grant funds awarded to the first author from the University of South Alabama (USA) Faculty Development Council and additional financial support was provided to the second author by the USA Honors Program and the University Committee on Undergraduate Research. We gratefully acknowledge the time and commitment of the participants who enrolled in this study. Two graduate students, Fran Battles and Rebekah Welty, assisted with data collection and diligently performed waveform analyses. The results of this study were presented at the $2014 \mathrm{HEAL}$ Conference in Lake Como, Italy, and at the 2015 ENT/SAAA/ SASLHA Congress in Durban, South Africa.

\section{REFERENCES}

Avila-Tang E, Elf JL, Cummings KM, Fong GT, Hovell MF, Klein JD, McMillen R, Winickoff JP, Samet JM. (2013) Assessing secondhand smoke exposure with reported measures. Tob Control 22(3):156-163.

Bear MF, Connors BW, Paradiso MA. (2001) Neuroscience: Exploring the Brain. Baltimore, MD: Lippincott Williams \& Wilkins.

Cruickshanks KJ, Klein R, Klein BE, Wiley TL, Nondahl DM, Tweed TS. (1998) Cigarette smoking and hearing loss: the epidemiology of hearing loss study. JAMA 279(21):1715-1719.

Etter JF, Vu Duc T, Perneger TV. (2000) Saliva cotinine levels in smokers and nonsmokers. Am J Epidemiol 151(3):251-258.

Fabry DA, Davila EP, Arheart KL, Serdar B, Dietz NA, Bandiera FC, Lee DJ. (2011) Secondhand smoke exposure and the risk of hearing loss. Tob Control 20(1):82-85.

Golub MS, Slotkin TA, Tarantal AF, Pinkerton KE. (2007) Visual recognition memory and auditory brainstem response in infant rhesus monkeys exposed perinatally to environmental tobacco smoke. Brain Res 1151:102-106.

Gopal KV, Herrington R, Pearce J. (2009) Analysis of auditory measures in normal hearing young male adult cigarette smokers using multiple variable selection methods with predictive validation assessments. Int $J$ Otol 1-7.

Hall JW. (2007) New Handbook of Auditory Evoked Responses. Boston, MA: Allyn and Bacon.

Harkrider AW, Champlin CA, McFadden D. (2001) Acute effect of nicotine on non-smokers: I. OAEs and ABRs. Hear Res 160(1-2): $73-88$.

Hashimoto I. (1982) Auditory evoked potentials from the human midbrain: slow brain stem responses. Electroencephalogr Clin Neurophysiol 53(6):652-657.

Jasper HH. (1958) The ten-twenty electrode system of the International Federation. Electroencephalogr Clin Neurophysiol 10: $371-375$

Jones LL, Hassanien A, Cook DG, Britton J, Leonardi-Bee J. (2012) Parental smoking and the risk of middle ear disease in children: a systematic review and meta-analysis. Arch Pediatr Adolesc Med 166(1):18-27.

Kraus N, McGee T, Stein L. (1994) The auditory middle latency response: clinical uses, development, and generating system. In: Jacobson JT, ed. Principles and applications in auditory evoked potentials. Needham Heights, MA: Allyn \& Bacon.

Le Houezec J, Benowitz NL. (1991) Basic and clinical psychopharmacology of nicotine. Clin Chest Med 12(4):681-699.

Lalwani AK, Liu YH, Weitzman M. (2011) Secondhand smoke and sensorineural hearing loss in adolescents. Arch Otolaryngol Head Neck Surg 137(7):655-662.

Lyons RA. (1992) Passive smoking and hearing loss in infants. Ir Med J 85(3):111-112.

Metzger KL, Maxwell CR, Liang Y, Siegel SJ. (2007) Effects of nicotine vary across two auditory evoked potentials in the mouse. Biol Psychiatry 61(1):23-30.

Moller AR. (1994) Neural generators of auditory evoked potentials. In: Jacobson JT, ed. Principles and applications of auditory evoked potentials. Needham Heights, MA: Allyn and Bacon. 
Panzano VC, Wayne GF, Pickworth WB, Connolly GN. (2010) Human electroencephalography and the tobacco industry: a review of internal documents. Tob Control 19(2):153-159.

Ramkissoon I, Beverly BL. (2014) The effects of age and stimulus type on auditory evoked potentials in cigarette smokers and nonsmokers. J Speech Lang Hear Res 57(1):271-284.

Ramkissoon I, Chambers RD. (2008) Effects of chronic and acute smoking on AMLRs in older and younger listeners. Int J Audiol 47(12):715-723.
U.S. Department of Health and Human Services (USDHHS). (2006) The Health Consequences of Involuntary Exposure to Tobacco Smoke: A Report of the Surgeon General. Atlanta, GA: U.S. Department of Health and Human Services, Centers for Disease Control and Prevention, Coordinating Center for Health Promotion, National Center for Chronic Disease Prevention and Health Promotion, Office on Smoking and Health.

World Health Organization International Agency for Research on Cancer. (2004) Tobacco smoke and involuntary smoking. IARC Monogr Eval Carcinog Risks Hum 83:1-1438. 